\title{
Microscopic Version of the Bohr-Mottelson Model and Its Application
}

\author{
H.G. Ganev \\ Joint Institute for Nuclear Research, Dubna, Russia \\ Received 4 October 2021 \\ doi: https://doi.org/10.55318/bgjp.2021.48.5-6.421
}

\begin{abstract}
The shell-model coupling scheme of the proton-neutron symplectic model (PNSM), defined by the following dynamical symmetry chain $S p(12, R) \supset S U(1,1) \otimes S O(6) \supset U(1) \otimes S U_{p n}(3) \otimes$ $S O(2) \supset S O(3)$, is considered. It is shown that it corresponds to a microscopic version of the Bohr-Mottelson collective model which captures the original relationships between its exactly solvable submodel limits. This variant of the PNSM provides an interesting and relevant shell-model symplectic-based framework for exploring the nuclear collective dynamics. Some simple applications of the present theory to different nuclei with various collective properties are given.
\end{abstract}

KEY WORDS: Bohr-Mottelson model, proton-neutron symplectic model, $\operatorname{Sp}(12, R)$ dynamical algebra.

\section{Introduction}

It is well known that in nuclear physics there are two fundamental models of nuclear structure that have been awarded with Nobel Prizes. The first one is the Bohr-Mottelson (BM) collective model [1] which is based on the quantization of the classical picture of surface vibrations and rotations. It has influenced all collective models because it provides the basic concepts and language in terms of which the nuclear collective motion can be described. It has demonstrated that the low-lying nuclear states can be described by considering only few macroscopic collective degrees of freedom when the intrinsic excitations lie high in energy. The second model is the shell model [2,3] which includes all manyfermion degrees of freedom. It provides a general microscopic framework in terms of which the other collective models can be founded and expressed.

The problem of giving the BM model a microscopic foundation have been realized long time ago. Its solution, however, was given through the algebraic approach by embedding it in the nuclear shell model. It was shown (see, e.g. $[4,5])$ 
that the collective model of Bohr and Mottelson admits a microscopic realization first by augmenting it by vorticity degrees of freedom, important for the appearance of low-lying collective states, and second by making it compatible with the composite many-fermion structure of the nucleus. The result is the onecomponent $S p(6, R)$ symplectic model [6] of nuclear collective motion, sometimes called a microscopic collective model, which is a submodel of the nuclear shell model. The $S p(6, R)$ model of nuclear rotations, among its submodels, contains the rigid-rotor model [7] and the Elliott's $S U(3)$ shell model of collective rotations [8], which obviously can be associated only with the rotor model limit of the BM model. The presence of vorticity in the $S p(6, R)$ model results in a complete range of possible collective flows from irrotational-flow (zero vorticity) to rigid rotations. This is of significant importance as well as the fact that the vortex-spin degrees of freedom are responsible for the appearance of lowlying collective states [4,5]. However, the $S p(6, R)$ model does not contain an $S O(5)$ or $S O(6)$ structure, which could allow to associate it with the $\beta$-rigid or $\beta$-soft but $\gamma$-unstable type dynamics of the Wilets-Jean (WJ) [9] model, in a manner similar, e.g., to that of IBM [10].

Recently, the generalized Bohr-Mottelson model was embedded in the twocomponent proton-neutron microscopic shell-model theory of the nucleus [11] within the framework of the proton-neutron symplectic model (PNSM) [12,13], providing in this way a more natural interpretation of the underlying BM quadrupole-monopole collective dynamics. The new embedding is performed by considering the shell-model coupling scheme of the PNSM, defined by the following dynamical symmetry chain $S p(12, R) \supset S U(1,1) \otimes S O(6) \supset$ $U(1) \otimes S U_{p n}(3) \otimes S O(2) \supset S O(3)$ [11]. We will demonstrate here that, in contrast to the $S p(6, R)$ symplectic model and the popular IBM [10], the presently considered microscopic version of the BM model has exactly solvable limits that have a relationship, closely resembling the one between the original $\gamma$-unstable WJ [9] and rigid-rotor [7, 14] submodels.

\section{Bohr-Mottelson Model and Its Exactly Solvable Limits}

The BM model can be formulated in algebraic terms by means of different spectrum generating algebras (SGA) and dynamical groups, which allows easily to perform an analysis at the algebraic level and to establish a relationship with other collective models of interest. The building blocks of the BM model are provided by the position $\alpha_{\mu}$ and momentum $\pi^{\nu}$ coordinates, which together with the identity operator $I$ close the Lie algebra of Heisenberg-Weyl group $H W(5)=\left\{\alpha_{\mu}, \pi^{\nu}, I\right\}$. In terms of quadrupole phonon operators, one can use the alternative realization $H W(5)=\left\{d_{\mu}^{\dagger}, d_{\nu}, I\right\}$. Different dynamical groups and SGA can be constructed from these coordinates. For our purposes, the following two dynamical groups $[H W(5)] U(5)$ and $S U(1,1) \otimes S O(5)$ [15] are of interest. It has been shown $[15,16]$ that the three dynamical subgroups $U(5)$, 
$\left[R^{5}\right] S O(5)$ and $\left[R^{5}\right] S O(3)$ correspond to the spherical vibrator, $\gamma$-unstable Wilets-Jean [9] and rigid-rotor [7, 14] exactly solvable limits of the BM model, respectively.

\subsection{Harmonic spherical vibrator}

The states in this limit are classified by the states of the five-dimensional harmonic oscillator, defined by the reduction chain:

$$
[H W(5)] U(5) \supset U(5) \supset S O(5) \supset S O(3),
$$

where $[H W(5)] U(5)$ is the semi-direct product group of Heisenberg-Weyl group, $H W(5)$ and $U(5)=\left\{d_{\mu}^{\dagger} d_{\nu}\right\}$, the latter being the symmetry group of the oscillator. Then for the HO Hamiltonian $H_{H V}=\sum_{\mu}\left(d_{\mu}^{\dagger} d_{\mu}+\frac{5}{2}\right)$ one immediately obtains the energies given by $E_{N}=(N+5 / 2) \hbar \omega$. The components of $E 2$ transition operator $T^{E 2} \simeq \alpha_{\mu}$ are provided by the generators of Abelian subgroup $R^{5} \equiv\left\{\alpha_{\mu}=\frac{1}{\sqrt{2}}\left(d_{\mu}^{\dagger}+d_{\mu}\right) ;\left[\alpha_{\mu}, \alpha_{\nu}\right]=0\right\}$ of $H W(5)$.

\subsection{Wilets-Jean model}

The dynamical subgroup chain of the $\gamma$-unstable $\beta$-rigid WJ model is [16]:

$$
[H W(5)] U(5) \supset\left[R^{5}\right] S O(5) \supset S O(5) \supset S O(3),
$$

where $\left[R^{5}\right] S O(5)$ is the semi-direct product group of $R^{5}$ and $S O(5)=\left\{\Lambda_{\mu \nu}=\right.$ $\left.-i\left(d_{\mu}^{\dagger} d_{\nu}-d_{\nu}^{\dagger} d_{\mu}\right)\right\}$. The irreducible representations of $\left[R^{5}\right] S O(5)$ are characterized by the rigid values of $\beta=\beta_{0}$. Hence, there is a problem with the deltafunction nature of the $\beta$ wave functions, which in turn don't have a convergent expansion in terms of the harmonic oscillator $U(5)$ states.

The $E 2$ transition operator $T^{E 2} \simeq \alpha_{\mu}$ in the $\mathrm{WJ}$ model belongs to $R^{5}$, while the Hamiltonian is expressed as a linear combination of the second-order Casimir operator of $S O(5)$, i.e. $H_{W J}=A^{\prime} C_{2}[S O(5)]=A^{\prime} \Lambda^{2}$. It eigenvalues are then given in terms of the $S O(5)$ quantum number $\tau$ by $E_{\tau}=A^{\prime} \tau(\tau+3)$. The yrast levels having $L=2 \tau$ produce a characteristic ratio $E_{4_{1}^{+}} / E_{2_{1}^{+}}=2.50$ of the WJ $\gamma$-unstable model for the first $2^{+}$and $4^{+}$states.

\subsection{Rigid rotor model}

The dynamical subgroup chain of the $\beta$-rigid and $\gamma$-rigid rotor model is [16]:

$$
[H W(5)] U(5) \supset\left[R^{5}\right] S O(5) \supset\left[R^{5}\right] S O(3) \supset S O(3),
$$

where $\operatorname{ROT}(3) \equiv\left[R^{5}\right] S O(3)=\left\{L_{k}, \alpha_{\mu} \mid\left[\alpha_{\mu}, \alpha_{\nu}\right]=0\right\}$ is the rigid-rotor model group of Ui [7]. The irreducible representations of the $R O T(3)$ group are characterized by both $\beta$-rigid and $\gamma$-rigid values. Looking at Eqs.(2) and (3), 
it follows that the $\beta$-rigid $\gamma$-rigid rotor model is a submodel of the $\mathrm{WJ} \beta$-rigid but $\gamma$-unstable model since the $\left[R^{5}\right] S O(3)$ is a subgroup of $\left[R^{5}\right] S O(5)$. This relationship between the two submodels has not been widely exploited in the literature. Hence, in the BM rigid-rotor submodel again there is a problem with the wave functions which are delta functions in both $\beta$ and $\gamma$.

Exact solution exists for the case of an axially-symmetric rotor. The rigid rotor Hamiltonian $H_{r o t}=\sum_{k=1}^{3} \frac{\hbar^{2} \bar{L}_{k}^{2}}{2 J_{k}}$ for this case becomes $H_{\text {rot }}=\frac{\hbar^{2} L^{2}}{2 J_{1}}+\left(\frac{\hbar^{2}}{2 J_{3}}-\right.$ $\left.\frac{\hbar^{2}}{2 J_{1}}\right) \bar{L}_{3}^{2}$, which eigenvalues are given by $E_{K L}=\frac{\hbar^{2}}{2 J_{1}} L(L+1)+\left(\frac{\hbar^{2}}{2 J_{3}}-\frac{\hbar^{2}}{2 J_{1}}\right) K^{2}$. The set $\left\{\bar{L}_{k}\right\}$ labels the intrinsic $S O(3)$ angular momentum operators, $J_{k}=$ $4 \mathfrak{B} \beta_{0}^{2} \sin ^{2}\left(\gamma_{0}-2 \pi k / 3\right)$ are the irrotational-flow moments of inertia, and $K$ is the third projection of the angular momentum operator on the body-fixed axis 3. Usually, in the numerical applications, the moments of inertia $J_{k}$ are treated as free parameters that are fitted to the experimental data. In this way, we see that the Hamiltonian of an axially-symmetric rotor is expressed in terms of the Casimir operators in the chain $S O(3) \supset S O(2)$. The $E 2$ transition operator $T^{E 2} \simeq \alpha_{\mu}$ is an element of the rigid-rotor algebra since $\left[R^{5}\right] S O(3)=\left\{L_{k}, \alpha_{\mu}\right\}$. Its matrix elements are proportional to the ordinary $S O(3)$ Clebsch-Gordan coefficients.

\subsection{Algebraic collective model}

Although the last two BM limiting cases just considered are characterized by dynamical subgroup chains, they are not particularly useful for the construction of basis states in which to diagonalize more general collective Hamiltonians, as this is done in the case of the five-dimensional oscillator. This is because the wave functions which diagonalize the $\left[R^{5}\right] S O(5)$ and $\left[R^{5}\right] S O(3)$ subgroups are not square-integrable. They contain factors which are delta functions in $\beta$ and $\gamma$. This limitation expresses the fact that rigidly-defined intrinsic quadrupole moments are unphysical and incompatible with the quantum mechanics. The resolution of this problem in the ACM is obtained by relaxing the $\beta$-rigidity of WJ model by replacing its dynamical group $\left[R^{5}\right] S O(5)$ with $S U(1,1) \otimes$ $S O(5)$, which results in a more physical collective model. Thus, in the ACM the following dynamical symmetry chain is used to define a continuous set of basis states for the BM model $[16,17]$ :

$$
\begin{array}{cccccccc}
S U(1,1) & S O(5) & \supset & U(1) \otimes & S O(3) & \supset & S O(2), \\
\lambda_{v} & v & \alpha & n & L & M
\end{array}
$$

where $S U(1,1)$ is a dynamical group for radial $\beta$ wave functions and $S O(5)$ group determines the angular part $(S O(5)$ spherical harmonics) that is characterized by the seniority quantum number $v$. An important characteristic of the ACM is that it enables $\beta$-rigid and $\gamma$-rigid limits to be approached in a continuous way with increasingly narrow but nevertheless square-integrable $\beta$ and $\gamma$ 
wave functions. The WJ and rigid-rotor submodels of the BM model are then seen as special cases of the more physical ACM. Additionally, the energies in the WJ and harmonic vibrator limits can be written as

$$
E_{W J}=A v(v+3)
$$

and

$$
E(n, v)=\left(2 n+v+\frac{5}{2}\right) \hbar \omega,
$$

respectively.

\section{PNSM Shell-Model Classification of the Collective States}

In the present work, we classify the shell-model nuclear states within the PNSM by the following reduction chain [11]:

$$
\begin{array}{cccccccccc}
S p(12, R) & \supset S U(1,1) \otimes S O(6) & \supset & U(1) & S U_{p n}(3) \otimes & S O(2) & \supset & S O(3), \\
\langle\sigma\rangle & \lambda_{v} & v & p & (\lambda, \mu) & \nu & q & L
\end{array}
$$

where bellow the different subgroups are given the quantum numbers that characterize their irreducible representations. The basis functions along the chain (7) can thus be written in the form [11]:

$$
\Psi_{\lambda_{v} p ; v \nu q L M}\left(r, \Omega_{5}\right)=R_{p}^{\lambda_{v}}(r) Y_{\nu q L M}^{v}\left(\Omega_{5}\right),
$$

where $Y_{\nu q L M}^{v}\left(\Omega_{5}\right)$ are the $\mathrm{SO}(6)$ Dragt's spherical harmonics $[18,19]$. The $S U(1,1)$ is a dynamical group for radial wave functions and $S O(6)$ group determines the angular part $(S O(6)$ spherical harmonics) that is characterized by the seniority quantum number $v$.

The $S U(1,1)$ algebra has unitary representations with orthonormal basis states $\left\{\left|\lambda_{v}, p\right\rangle ; p=0,1,2, \ldots\right\}$ that are defined by the equations [20]:

$$
\begin{aligned}
S_{+}^{\left(\lambda_{v}\right)}\left|\lambda_{v}, p\right\rangle & =\sqrt{\left(2 \lambda_{v}+p\right)(p+1)}\left|\lambda_{v}, p+1\right\rangle, \\
S_{-}^{\left(\lambda_{v}\right)}\left|\lambda_{v}, p\right\rangle & =\sqrt{\left(\lambda_{v}-1+p\right) p}\left|\lambda_{v}, p-1\right\rangle, \\
S_{0}^{\left(\lambda_{v}\right)}\left|\lambda_{v}, p\right\rangle & =\frac{1}{2}\left(\lambda_{v}+2 p\right)\left|\lambda_{v}, p\right\rangle,
\end{aligned}
$$

for any value of $\lambda_{v}\left(\lambda_{v}>1\right)$. Thus, e.g., for the harmonic oscillator series representations of $S U(1,1)$ we have $\lambda_{v}=v+6 / 2$. From Eq.(11) it then follows $E_{p v}=\left(2 p+\lambda_{v}\right) \hbar \omega=(2 p+v+6 / 2) \hbar \omega$ to be compared with Eq.(6). For $\lambda_{v}>v+6 / 2$, the radial wave functions are eigenfunctions of a Hamiltonian with a potential $[21,22]$

$$
V^{\left(\lambda_{v}\right)}(r)=\frac{\left(\lambda_{v}-1\right)^{2}-4}{2 r^{2}}+\frac{r^{2}}{2}
$$


which corresponds to the addition of a centrifugal-like potential to the harmonic oscillator potential. In this case, $\lambda_{v}=1+\sqrt{(v+4 / 2)^{2}+\left(r_{0}\right)^{4}}$ and one obtains the so-called modified oscillator $S U(1,1)$ irreps [21]. The energies for the Davidson oscillator $E_{p v}=\left(2 p+\lambda_{v}\right) \hbar \omega=\left(2 p+1+\sqrt{(v+4 / 2)^{2}+\left(r_{0}\right)^{4}}\right) \hbar \omega$ for large values of $r_{0}$ can then be expanded in inverse powers of $r_{0}$ to give

$$
E_{p v}=E_{0}+2 p \hbar \omega+A v(v+4)+\ldots
$$

where $A=\hbar \omega / 2 r_{0}^{2}$. For $r_{0} \rightarrow \infty$ and $\hbar \omega \rightarrow \infty$ this expression corresponds to the microscopic counterpart of the $\beta$-rigid, $\gamma$-unstable WJ model with energies given by $E_{v}=E_{0}+A v(v+4)$. Note that, in contrast to $\mathrm{WJ}$ model value 2.50, this expression gives a characteristic ratio $E_{4_{1}^{+}} / E_{2_{1}^{+}} \simeq 2.67$ of the ground state band energies, for which $L=v$ (see the left diagonal of Table 1 of Ref. [11] with $(\lambda, \mu)=(k, 0), k=0,2,4, \ldots)$.

A microscopic analogue of the rigid rotor is provided by the $S U_{p n}(3)$ structure, which for large dimensional $S U(3)$ representations contracts [23] to $\left[R^{5}\right] S O(3)$ of $\mathrm{Ui}$ and the rigid rotor model states are approached.

The results just obtained can alternatively be achieved in a pure algebraic way by taking the proper Casimir operators in the Hamiltonian, as it is done in the next section.

\section{Application}

Applications within the framework of the algebraic approach to nuclear structure vary according to the type of nuclear interaction that is used. Initially, the simplest case of using schematic or algebraic interactions has been widely exploited by many authors. But with the increase of the high-performance computing technologies during the last decades, different computationally intensive large-scale calculations that use modern high-precision realistic interactions inspired from the QCD became possible, like those performed within the nocore shell model (NCSM) [24] or symmetry-adapted no-core shell model (SANCSM) [25] frameworks. Here, however, we use a simple algebraic interaction.

A general dynamical symmetry Hamiltonian can be written as a linear combination of the Casimir operators of different subgroups of the chain (7):

$$
\begin{aligned}
H=H\left(S_{0}^{\left(\lambda_{v}\right)}, S_{+}^{\left(\lambda_{v}\right)}, S_{-}^{\left(\lambda_{v}\right)}\right) & +V(r) \\
+ & A \Lambda^{2}+f\left(C_{2}\left[S U_{p n}(3)\right], C_{2}[S O(3)]\right)
\end{aligned}
$$

where $\left\{S_{0}^{\left(\lambda_{v}\right)}, S_{+}^{\left(\lambda_{v}\right)}, S_{-}^{\left(\lambda_{v}\right)}\right\}$ are the generators of the group $S U(1,1)$ and $\Lambda^{2}=$ $C_{2}[S O(6)]$. The starting point of the present application is the following dy- 
namical symmetry Hamiltonian

$$
\begin{aligned}
H=n \hbar \omega+A \Lambda^{2}+B C_{2}[ & \left.S U_{p n}(3)\right] \\
& +a C_{2}[S O(3)]+b K^{2}+c\left(C_{2}[S O(3)]\right)^{2},
\end{aligned}
$$

where we have used the fact that $H_{0}=2 S_{0}^{(\lambda)}=n \hbar \omega$ represents the harmonic oscillator mean field. The last three terms represent a residual rotor part which takes into account the band characteristics, like the observed moment of inertia, the $K$-band splitting and the centrifugal stretching effects. Additionally, we consider the following simple Hamiltonian [20]:

$$
H_{h m i x}=h\left(G^{2}(a, a) \cdot F^{2}(b, b)+G^{2}(b, b) \cdot F^{2}(a, a)\right),
$$

which mixes different $S U_{p n}(3)$ multiplets within the maximal seniority $S O(6)$ representation $v_{0}$ contained in the corresponding symplectic bandhead. For more information we refer the reader to Ref. [20], where the required matrix elements are also given.

We consider the following three nuclei ${ }^{158} \mathrm{Gd},{ }^{150} \mathrm{Nd}$ and ${ }^{148} \mathrm{Nd}$ with the characteristic ratios [26] $E_{4_{1}^{+}} / E_{2_{1}^{+}} \simeq 3.26,2.93$, and 2.49, respectively. The relevant $\operatorname{Sp}(12, R)$ irreducible representation for each nucleus is determined by the lowest-weight $U(6)$ irrep, which in turn is fixed by the underlying protonneutron shell-model structure. Thus, according to the shell-model considerations based on the pseudo- $S U(3)$ scheme [27], we choose the following $S p(12, R)$ irreducible representations: $0 \mathrm{p}-0 \mathrm{~h}[36]_{6}$ for ${ }^{158} \mathrm{Gd}, 0 \mathrm{p}-0 \mathrm{~h}[24]_{6}$ for ${ }^{150} \mathrm{Nd}$, and $0 \mathrm{p}-0 \mathrm{~h}[18]_{6}$ for ${ }^{148} \mathrm{Nd}$, respectively. We diagonalize the model Hamiltonian in the irreducible collective space spanned by the the maximal seniority $S O(6)$ representation $v_{0}$, i.e. $v_{0}=36\left({ }^{158} \mathrm{Gd}\right), v_{0}=24\left({ }^{150} \mathrm{Nd}\right)$, and $v_{0}=18\left({ }^{148} \mathrm{Nd}\right)$, correspondingly, of the symplectic bandhead for each nucleus. Hence the first two terms in the Hamiltonian (15) are irrelevant and can be dropped. In addition, due to the prolate-oblate symmetry of the $S U_{p n}(3)$ multiplets related with the conjugate $\mathrm{SU}_{p n}(3)$ multiplets $(\lambda, \mu)$ and $(\mu, \lambda)$ contained within the corresponding $\mathrm{SO}(6)$ irreducible representations, we use only the $\mathrm{SU}(3)$ multiplets $(\lambda, \mu)$ with $\lambda \geq \mu$. The results of diagonalization for the low-lying excitation spectra in ${ }^{158} \mathrm{Gd},{ }^{150} \mathrm{Nd}$, and ${ }^{148} \mathrm{Nd}$ together with the experimental data are shown in Figure 1, while the intraband $B(E 2)$ transition strengths between the states of the ground band for these three nuclei are given in Figure 2. In the calculation of the corresponding $B(E 2)$ values, no effective charges are used. The values of the model parameters (in $\mathrm{MeV}$ ) are as follows: $B=-0.039, a=0, b=0.247$, $c=0$, and $h=-0.147$ for ${ }^{158} \mathrm{Gd} ; B=-0.046, a=0.015, b=0.075$, $c=-0.000016$ and $h=-0.074$ for ${ }^{150} \mathrm{Nd} ; B=-0.038, a=0.023$, $b=0.203, c=-0.000055$ and $h=-0.0924$ for ${ }^{148} \mathrm{Nd}$. We see a good description of the experimental data for all the three nuclei under consideration. Note also that the excitation spectra of ${ }^{158} \mathrm{Gd}$ is obtained without using an adjustable moment of inertia. 

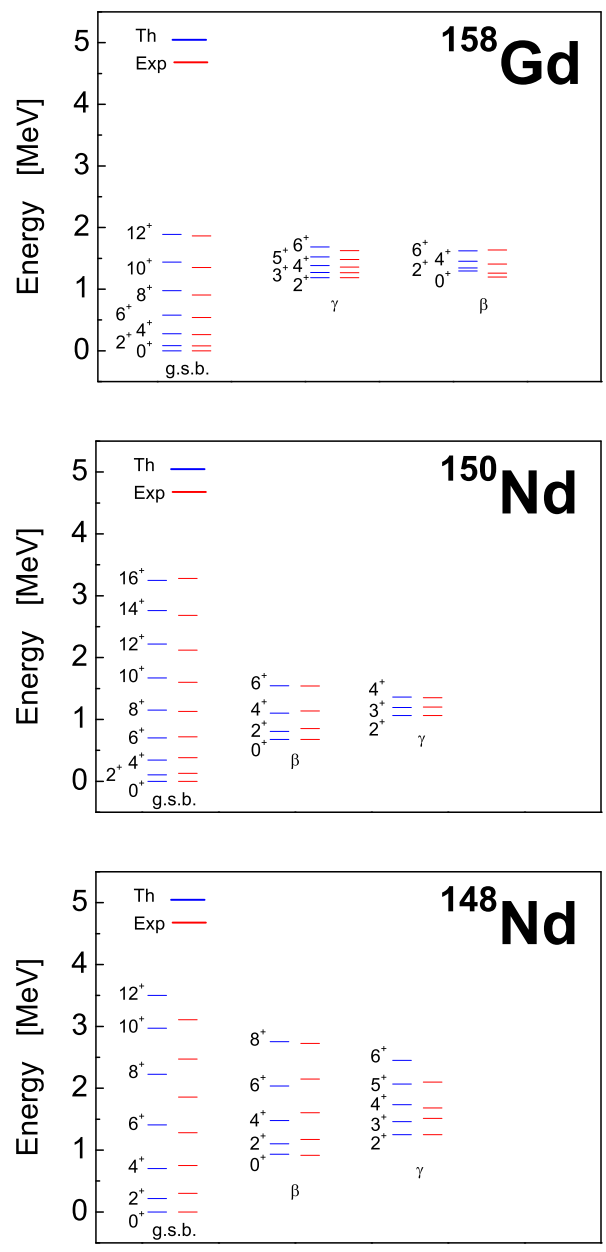

Figure 1. (Color online) Comparison of experimental energy levels with the theory for the low-lying ground, $\beta$ and $\gamma$ bands in ${ }^{158} \mathrm{Gd},{ }^{150} \mathrm{Nd}$, and ${ }^{148} \mathrm{Nd}$.

\section{Conclusions}

Microscopic analogues of the exactly solvable limits of the Bohr-Mottelson collective model are shortly considered in respect to the original BM submodels. Microscopic considerations are given within the framework of the PNSM, in which the relevant shell-model coupling scheme is defined by the following dynamical symmetry chain $S p(12, R) \supset S U(1,1) \otimes S O(6) \supset U(1) \otimes S U_{p n}(3) \otimes$ $S O(2) \supset S O(3)$, according to which the many-particle nuclear shell-model states are classified. We note that within the present approach we obtain the proper relationships between the original BM submodels, especially that be- 
Microscopic Version of the Bohr-Mottelson Model and Its Application
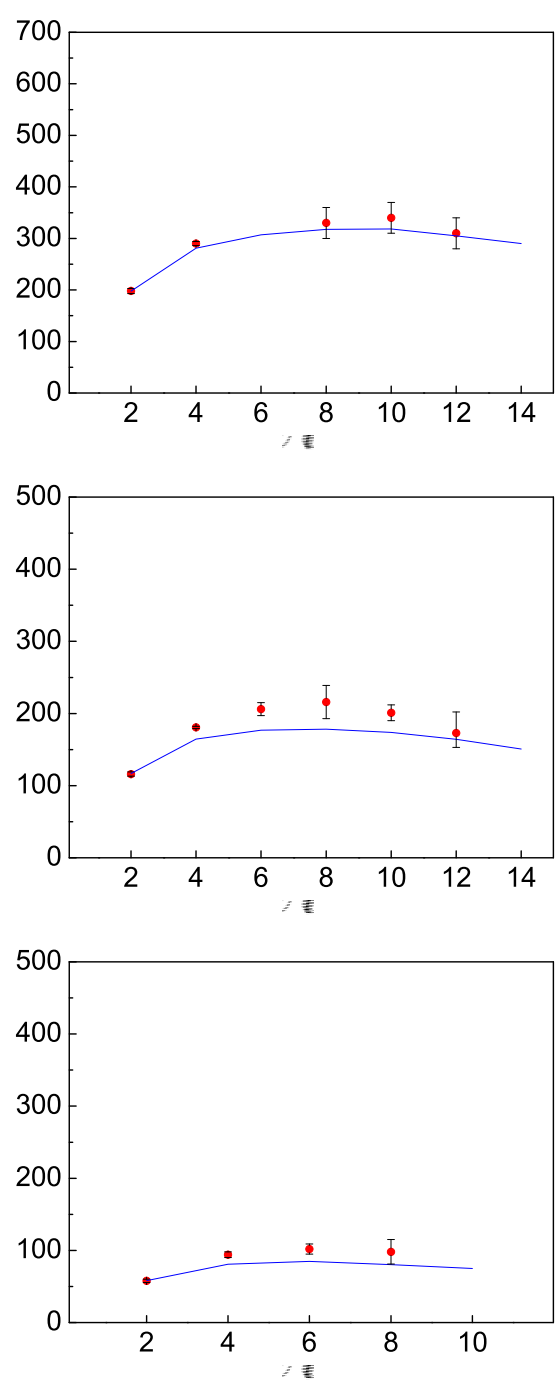

Figure 2. (Color online) Calculated and experimental intraband $B(E 2)$ values in Weisskopf units between the states of the ground band in ${ }^{158} \mathrm{Gd},{ }^{150} \mathrm{Nd}$, and ${ }^{148} \mathrm{Nd}$. No effective charge is used.

tween the $\gamma$-unstable Wilets-Jean and rigid rotor models. This is in contrast to other phenomenological and microscopic approaches aiming the microscopic foundation of the BM model. This fact was stressed in Ref. [28] and was a central point in our considerations. In some respects, the present considerations also resemble those performed in the ACM. However, the main difference 
between the present approach and the ACM is in the irreducible collective subspaces in which the model Hamiltonians act. For the microscopic models, like the PNSM, the state space is defined by allowed $O(A-1)$ (or complementary to it $S p(12, R)$ ) irreducible representations $\omega$ that are consistent with the Pauli principle, whereas for the phenomenological models the state space in which the collective Hamiltonians act is defined by the $O(A-1)$-scalar subspace of the many-particle Hilbert spaces with $\omega=(0)$. The specific structure of this violated permutational symmetry space $\mathbb{H}^{\omega=(0)}$ is that it gives a "deep freezing" of the microscopic collective features of the used Hamiltonians and make them similar to those in the Bohr-Mottelson theory, associated with the irrotationalflow collective dynamics. Thus, the combined proton-neutron dynamics in the present approach is governed by the microscopic shell-model intrinsic structure of the symplectic bandhead. To illustrate the present symplectic-based protonneutron shell-model approach, we apply the theory to three nuclei with different collective properties - namely, ${ }^{158} \mathrm{Gd},{ }^{150} \mathrm{Nd}$, and ${ }^{148} \mathrm{Nd}$. A good description of the excitation energies of the ground, $\beta$ and $\gamma$ bands, as well as for the ground state intraband $B(E 2)$ transition strengths is obtained for these three nuclei. The quadrupole collectivity is described without the use of an effective charge. More detailed calculations will be given elsewhere.

\section{References}

[1] A. Bohr and B. R. Mottelson (1975) “Nuclear Structure” (W.A. Benjamin Inc., New York) Vol. II.

[2] M.G. Mayer (1949) Phys. Rev. 751969.

[3] K. Heyde (1990) "The Nuclear Shell Model" (Springer-Verlag, Berlin, Heidelberg).

[4] D.J. Rowe (1985) Rep. Prog. Phys. 481419.

[5] D.J. Rowe (1996) Prog. Part. Nucl. Phys. 37265.

[6] G. Rosensteel, D.J. Rowe (1977) Phys. Rev. Lett. 3810.

[7] H. Ui (1970) Prog. Theor. Phys. 44153.

[8] J.P. Elliott(1958) Proc. R. Soc. A 245 128; 562.

[9] L. Wilets, M. Jean (1956) Phys. Rev. 102788.

[10] F. Iachello, A. Arima (1987) "The Interacting Boson Model" (Cambridge University Press, Cambridge).

[11] H.G. Ganev (2021) Eur. Phys. J. A 57181.

[12] H.G. Ganev (2014) Eur. Phys. J. A 50183.

[13] H.G. Ganev (2015) Eur. Phys. J. A 5184.

[14] A.S. Davydov, G.F. Filippov (1958) Nucl. Phys. 8237.

[15] D.J. Rowe, J.L. Wood (2010) "Fundamentals of Nuclear Models: Foundational Models" (World Scientific Publisher Press, Singapore).

[16] D.J. Rowe (2004) Nucl. Phys. A 735372.

[17] D.J. Rowe, P.S. Turner (2005) Nucl. Phys. A 75394.

[18] A.J. Dragt (1965) J. Math. Phys. 6533.

[19] E. Chacon, O. Castanos, A. Frank (1984) J. Math. Phys. 251442. 
Microscopic Version of the Bohr-Mottelson Model and Its Application

[20] H.G. Ganev (2021) Chin. Phys. C 45114101.

[21] D.J. Rowe (2005) J. Phys. A: Math. Gen. 3810181.

[22] D.J. Rowe (2006) In “Symmetry, Spectroscopy and SCHUR”, Eds. R.C. King, M. Bylicki, and J. Karwowski (N. Copernicus Univ. Press, Torun) pp. 241-249.

[23] R. Le Blanc, J. Carvalho, D.J. Rowe (1984) Phys. Lett. B 140155.

[24] P. Navratil, J.P. Vary, B.R. Barrett (2000) Phys. Rev. Lett. 845728.

[25] J.P. Draayer, T. Dytrych, K.D. Launey, D. Langr (2012) Prog. Part. Nucl. Phys. 67 516.

[26] National Nuclear Data Center (NNDC), http://www.nndc.bnl.gov/.

[27] J.P. Draayer, K.J. Weeks (1984) Ann. Phys. 15641.

[28] D.J. Rowe, G. Thiamova (2005) Nucl. Phys. A 76059. 